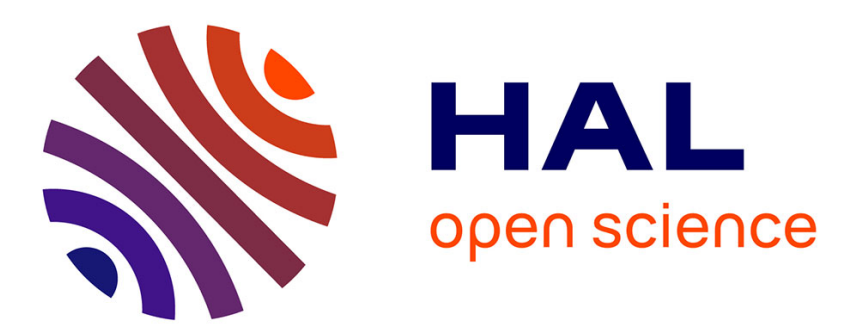

\title{
Creep Damage Coupled Effects: Experimental Investigation on Bending Beams with Various Sizes
}

\author{
Mirvat Omar, Ahmed Loukili, Gilles Pijaudier-Cabot, Yann Le Pape
}

\section{To cite this version:}

Mirvat Omar, Ahmed Loukili, Gilles Pijaudier-Cabot, Yann Le Pape. Creep Damage Coupled Effects: Experimental Investigation on Bending Beams with Various Sizes. Journal of Materials in Civil Engineering, 2009, 21 (2), pp.65-72. 10.1061/(ASCE)0899-1561(2009)21:2(65) . hal-01006708

\section{HAL Id: hal-01006708 https://hal.science/hal-01006708}

Submitted on 3 Jul 2017

HAL is a multi-disciplinary open access archive for the deposit and dissemination of scientific research documents, whether they are published or not. The documents may come from teaching and research institutions in France or abroad, or from public or private research centers.
L'archive ouverte pluridisciplinaire HAL, est destinée au dépôt et à la diffusion de documents scientifiques de niveau recherche, publiés ou non, émanant des établissements d'enseignement et de recherche français ou étrangers, des laboratoires publics ou privés. 


\title{
Creep-Damage Coupled Effects: Experimental Investigation on Bending Beams with Various Sizes
}

\author{
Mirvat Omar ${ }^{1}$; Ahmed Loukili ${ }^{2}$; Gilles Pijaudier-Cabot ${ }^{3}$; and Yann Le Pape
}

\begin{abstract}
The serviceability of concrete structures is a problem in which creep and damage are coupled. In this paper, we present experimental investigations on concrete that involve tertiary creep. We consider residual capacity tests on notched bending beams and investigate the evolution of size effect due to basic creep. In these experiments, beams are first subjected to a constant load, at a given ratio of the maximum capacity during 90 days. They are then loaded up to failure and their residual capacity is obtained. During the creep phase, acoustic emission is analyzed and it shows that damage develops under creep. The comparison between the size effect test results with and without creep exhibits variations of the fracture properties of the material. The fracture energy and the size of the fracture process zone decrease when creep occurs prior to failure. These results, which include size effect fracture tests, may serve for the development and validation of coupled creep-damage models.
\end{abstract}

Keywords: Creep; Cracking; Acoustic techniques; Concrete beams.

\section{Introduction}

In common practice, it is usually assumed that linear viscoelasticity takes place for low load levels and that in the same loading range, the instantaneous mechanical behavior of concrete remains elastic. For high load levels, deviation from linearity of the response of concrete to creep is expected and deviation from linearity of the instantaneous mechanical response occurs as well. Under high sustained loads, cracks grow and interact with the time dependent response of the bulk material. This is often denoted as "nonlinear creep".

Modeling nonlinear creep of concrete is of fundamental importance for severely loaded structures, as creep may decrease the material strength with increasing time. For some specific structures such as containment vessels, cooling towers or dams, the evaluation of their residual load carrying capacity is a problem of growing importance since it has an impact on their safety margin and life time. These structures are often exposed to high stresses for a long period of time, during which damage develops and may increase with time. The development of damage in itself may be small, not affecting the global stability of the structure in service conditions but still have an impact on the serviceability of the

${ }^{1}$ Institut de Recherche en Génie Civil et Mécanique GeM-UMR CNRS 6183, Ecole Centrale de Nantes, BP 92101, F-44321 Nantes cedex 3, France.

${ }^{2}$ Professor, Institut de Recherche en Génie Civil et Mécanique GeMUMR CNRS 6183, Ecole Centrale de Nantes, BP 92101, F-44321 Nantes cedex 3, France.

${ }^{3}$ Professor, Laboratoire des fluides complexes (UMR 5150), ISA BTP, 64600 Anglet, France.

${ }^{4}$ Research Engineer, Electricité de France, EDF R\&D, Les renardières, Moret sur Loing, France.

Note. Associate Editor: Nemkumar Banthia. Discussion open until July 1, 2009. Separate discussions must be submitted for individual papers. The manuscript for this paper was submitted for review and possible publication on November 1, 2006; approved on May 16, 2008. This paper is part of the Journal of Materials in Civil Engineering, Vol. 21, No. 2, February 1, 2009. CASCE, ISSN 0899-1561/2009/2-65-72/\$25.00. structure. The case of containment vessels is a typical example where damage may induce some increase of the permeability, affect the water or gas tightness, and, thus, may overcome some security threshold related to admissible leakage rates.

Therefore, the results arising from short time tests cannot be considered as a sufficient basis for assessing the safety of such structures and it is of great interest to devise methods for evaluating their residual capacity, and at the same time nonlinear creep. This problem has drawn the attention of some authors, among them Rüsch et al. (1958). They reported the first experiments where the effect of creep on the resistance and deflections of concrete specimens under compression tests was investigated. They found that the capacity of a structure subjected to creep loads appeared to be 70 to $80 \%$ of that observed in short time compression tests. Gettu and Bazant (1992) investigated the effect of the loading rate on the fracture properties of concrete. They carried out three-point bend tests on geometrically similar notched specimens and found that the fracture energy decreases with the loading rate. Theoretical explanations based on stress relaxation in the fracture process zone or energy barrier functions have been proposed as well (Bazant and Planas 1998). There are also in the literature, several constitutive models for nonlinear creep, e.g., based on damage mechanics coupled to linear viscoelasticity (Mazzotti and Savoia 2003; Omar et al. 2004), or smeared crack models coupled to linear viscoelasticity (de Borst et al. 1993). Elastoplastic models coupled to nonlinear creep can be conceived as well.

In the present paper, the main results of a set of experimental creep tests on concrete three-point bend specimens at different load levels are presented. The objective is twofold:

1. To investigate the ranges of variation of the time response due to creep-damage coupled effects under constant load. For each creep test, different load levels were applied, starting from a low level (36\% of peak load) where linear viscoelasticity applies, up to a high level ( $80 \%$ of peak load) where tertiary creep causing failure at a finite time is expected. Acoustic emission analysis was used in order to detect damage evolution during creep tests. 
Table 1. Concrete Mixture Proportions

\begin{tabular}{lc}
\hline Constituents & Dosage $\left(\mathrm{kg} / \mathrm{m}^{3}\right)$ \\
\hline Gravel & 784 \\
$12.5 / 25$ & \\
Gravel & 316 \\
G:/12.5 & \\
Sand & 772 \\
$0 / 4 \mathrm{~mm}$ & \\
Cement & 350 \\
CEMII 42.5 & \\
Filler nature & Limestone \\
Superplasticizer & 1.3 \\
Mass density & 2,360 \\
\hline
\end{tabular}

2. To evaluate the residual capacity of concrete after creep. We carried out for this purpose a series of tests on geometrically similar specimens, which had been subjected to creep beforehand. We then evaluated the variation of the fracture energy of the specimens.

In the following, we speak about damage instead of fracture and consider the effect of creep on fracture-or damage - growth. In our understanding and for concrete and other quasi-brittle materials, fracture (macrocracking) results from the growth and coalescence of microcracks, distributed in a fracture process zone. The two concepts are equivalent (Mazars and Pijaudier-Cabot 1996).

\section{Material Properties and Fracture Tests}

The experimental program aimed first at determining the mechanical characteristics (e.g., tensile strength, compressive strength, fracture energy) of the specific concrete used, before performing the next steps, flexural creep tests on prismatic bending beams and residual capacity tests.

All the specimens were made with a mix that consisted of ordinary portland cement CPA-CEMII 42.5, fine sand with a maximum size of $5 \mathrm{~mm}$, crushed gravel of size 5 to $25 \mathrm{~mm}$, a superplasticizer agent (Glenium 21), and water. Mix proportions are shown in Table 1 . This mixture is characterized by a watercement ratio of 0.56 and a slump of $40 \mathrm{~mm}$.

Three different types of tests were performed in order to determine the mechanical characteristics of concrete. Compressive strength $\mathrm{fc}$, tensile strength $\mathrm{ft}$, and elastic modulus were measured on $160 \times 320 \mathrm{~mm}$ cylinders at 28 days. Tensile strength was assessed through splitting tests. The dynamic modulus of elasticity Edyn was determined with a Grindosonic apparatus. This device gives the frequency of a vibration created by a slight shock on the sample and the elastic modulus is calculated from this frequency with the Spinner and Teft (1961) model. All tests were carried out on three samples, and mean values of mechanical properties are given in Table 2 .

The third kind of test consisted of a three-point bend size effect experiment on prismatic geometrically similar notched beams (Fig. 1). Three different sizes were used; the depths were $D 1=100 \mathrm{~mm}, D 2=200 \mathrm{~mm}$, and $D 3=400 \mathrm{~mm}$ with respective lengths 350, 700, and $1400 \mathrm{~mm}$, while the thickness was kept constant for all the specimens $b=100 \mathrm{~mm}$. The span to depth ratio was $l / D=3$ for all specimens. Each specimen had a notch of depth $0.15 D(a 0)$ and thickness $3 \mathrm{~mm}$ (same for all dimensions) at midspan on the bottom face, which was obtained by placing a
Table 2. Mechanical Properties of Concrete

\begin{tabular}{lc}
\hline Properties & $\begin{array}{c}\text { Mean values on three } \\
\text { specimens }\end{array}$ \\
\hline $\mathrm{f}_{\mathrm{c}}^{\prime}(\mathrm{MPa})$ & 41.5 \\
$\mathrm{f}_{\mathrm{t}}^{\prime}(\mathrm{MPa})$ & 3.5 \\
$\mathrm{E}_{\mathrm{dyn}}(\mathrm{MPa})$ & 39,000 \\
$\mathrm{D} 1: \mathrm{F}_{\max }(\mathrm{daN})$ & 863,4 \\
$\sigma_{\mathrm{N}}(\mathrm{MPa})$ & 5.61 \\
$\mathrm{D} 2: \mathrm{F}_{\max }(\mathrm{daN})$ & $1,401.1$ \\
$\sigma_{\mathrm{N}}(\mathrm{MPa})$ & 4.42 \\
$\mathrm{D} 3: \mathrm{F}_{\max }(\mathrm{daN})$ & $2,377.3$ \\
$\sigma_{\mathrm{N}}(\mathrm{MPa})$ & 4.81 \\
$\mathrm{G}_{\mathrm{f}}(\mathrm{N} / \mathrm{m})$ & 33.1 \\
\hline
\end{tabular}

plastic plate in the molds before casting. Nine prismatic specimens were cast, three for each size. Curing conditions for all specimens were 28 days at $50 \% \mathrm{RH}$ and controlled temperature of $20^{\circ} \mathrm{C}$.

Size effect tests followed the guidelines established by RILEM (1990) using a closed-loop testing machine (160 KN capacity INSTRON machine), under crack mouth opening displacement (CMOD) control. A general view of the experimental setup is provided in Fig. 2. Prior to the test, two steel plates with knife edges were glued on both sides of the notch. The clip gage used to measure the CMOD was then placed between the knife edges. In order to measure the deflection of a central point of the beam, a laser extensometer was used. This extensometer was placed at midspan on a steel beam, which was attached at both ends and at half-height of the concrete beam. A metallic plate glued on the specimen in front of the extensometer reflected the laser beam in order to measure the deflection. Deflection was measured with this setup so as not to include the effect of local deformations at the support and load points. A data acquisition system was used to record the load, the CMOD, and the deflection with time. Fig. 3 shows the average responses of the bending tests for all dimensions. Mean values of mechanical properties are summarized in Table 2.

Another set of three-point bending tests was carried out on beams of size $D 2$ : $100 \times 200 \times 700 \mathrm{~mm}$ without notches. These tests were aimed at determining the maximum load of unnotched beams in order to calibrate the applied load in the creep tests, where beams having the same geometry have been used. The average maximum load determined from flexural tests on three of these specimens is about $24.1 \mathrm{kN}$.

\section{Creep Tests}

The creep tests were performed on frames designed especially for this program and placed in a climate controlled chamber. The frames have a capacity ranging from 5 to $50 \mathrm{kN}$ and can accommodate geometrically similar specimens of three different sizes. The load is applied by gravity with a weight and counterweight system, which enables a fine tuning of the load. Fig. 4 shows a general view of these creep frames.

Two kinds of three-point bend creep tests were performed: (1) tests on unnotched specimens with exchanges of moisture between concrete and the climate controlled chamber; and (2) tests on notched specimens where the exchange of moisture was prevented. For these two types of tests, we are interested in the time dependent response of the beams. For convenience, the displace- 

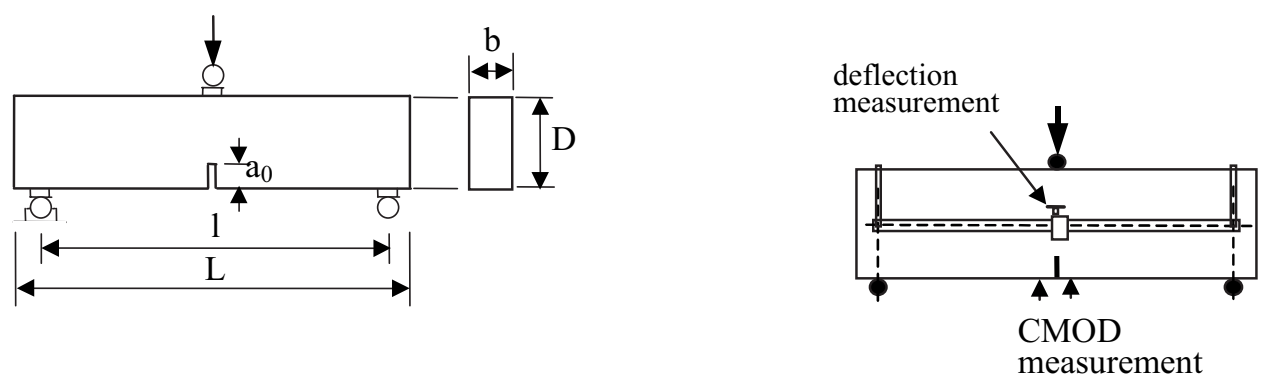

Fig. 1. Three-point bend experiments on notched specimen

ment at midspan is converted into a specific displacement, which is the amount of deflection per unit applied stress (this is the stress evaluated according to the beam theory, same as the nominal stress in the size effect law discussed later). After having subtracted the instantaneous response, the specific displacement creep remains. Note that this displacement creep is the outcome of a structural response. It is not a material characteristic but a structural one.

\section{Drying Creep Tests}

These tests were performed on four unnotched beams of dimension $D 2$ loaded at $50,60,70$, and $80 \%$ of the maximum load at $20^{\circ} \mathrm{C}$ and $50 \% \mathrm{RH}$. The specimens were not protected from exchanges of moisture with the testing room. Therefore, basic creep and drying creep occurred at the same time. The age of concrete at the beginning of the creep tests was 28 days. Fig. 5 presents the total displacement (creep displacement plus instantaneous displacement) measured on the specimens versus time for the first three loading levels. These curves indicate that creep kinetics are comparable for all loading levels, with the exception of the specimen loaded at $70 \%$ of the peak load, where failure due to tertiary creep occurs after 8 days. In this test, we observed an important acceleration of the creep kinetic and then failure. It is worth noting that the specimen loaded at $80 \%$ of peak load experienced total failure only 2 min after application of the load.

The data obtained for the highest load levels are typical of tertiary creep. They apply to relatively thin structures where the effect of drying creep on damage is much more important than

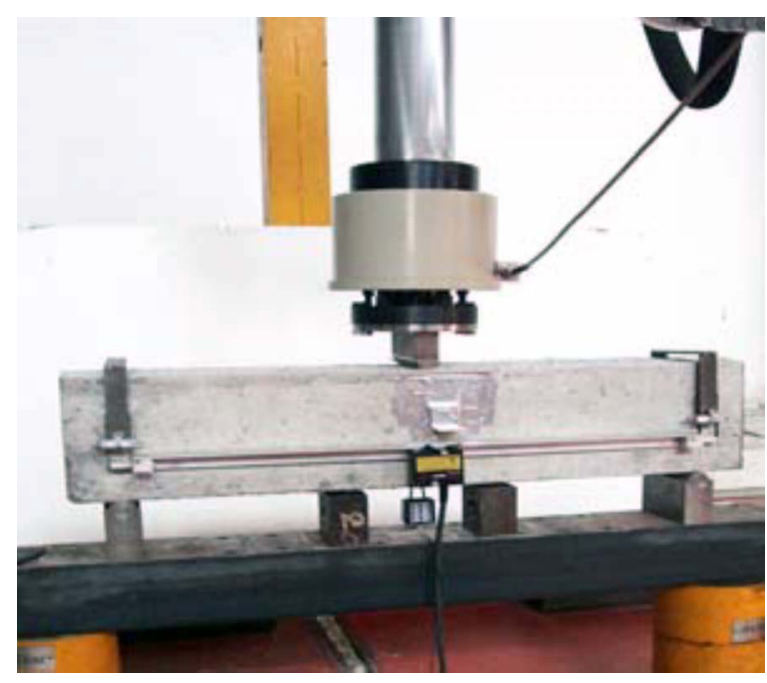

Fig. 2. General view of experimental fracture test setup that of basic creep. This is probably the reason why the creep kinetics does not depend on the load level, which is rather surprising. It is controlled by drying creep, which is the same for all the specimens since the curing and testing conditions are identical for all tests. For much more massive structures, which are of concern in the present study (nuclear vessels are typically more than $1 \mathrm{~m}$ thick, prestressed in between 12 and 18 months after casting, with a rather constant moisture environment), basic creep may be much more critical than drying creep. This is the reason why the remaining part of the experimental study focuses on the interaction between basic creep and damage.

\section{Basic Creep Tests}

Two kinds of basic creep tests were performed. The first one was intended to study the influence of the load level on creep. In the second kind of test, the effect of the size of the specimen, at a constant load level was investigated.

The first test series consisted of three notched beams of dimension $D 1$ loaded at 80,60 , and $36 \%$ of the maximum load recorded under monotonic loading in the material characterization study. Specimens were protected from desiccation by a double layer of self-adhesive aluminium paper. Hence, basic creep was considered only. The experimental measurement of basic creep of concrete requires also drying shrinkage to be prevented (Khelidj et al. 1998). The curing conditions ( 3 months at $100 \% \mathrm{RH}$ and $20^{\circ} \mathrm{C}$ ) guaranteed to avoid autogeneous shrinkage so that basic creep

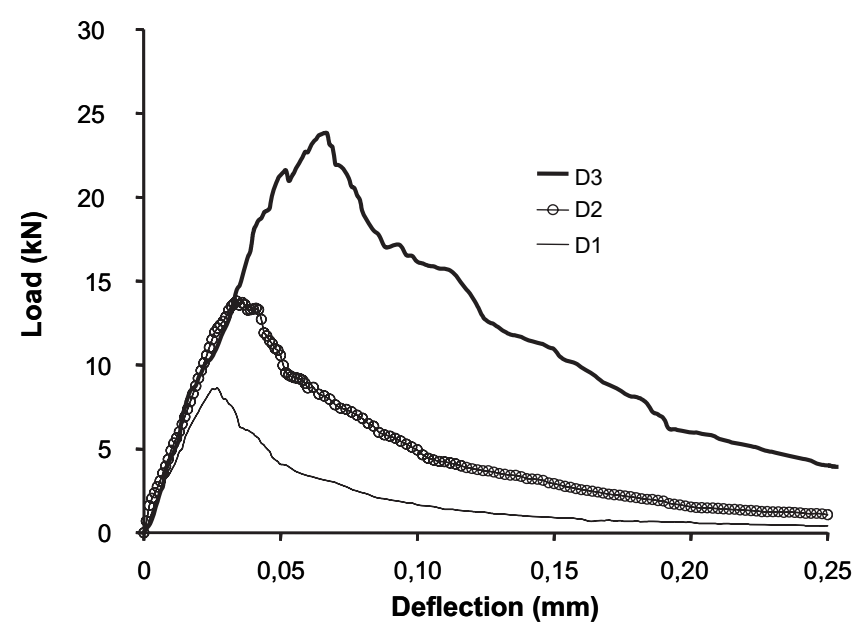

Fig. 3. Average load-deflection curves for small (D1), medium (D2), and large (D3) sizes 


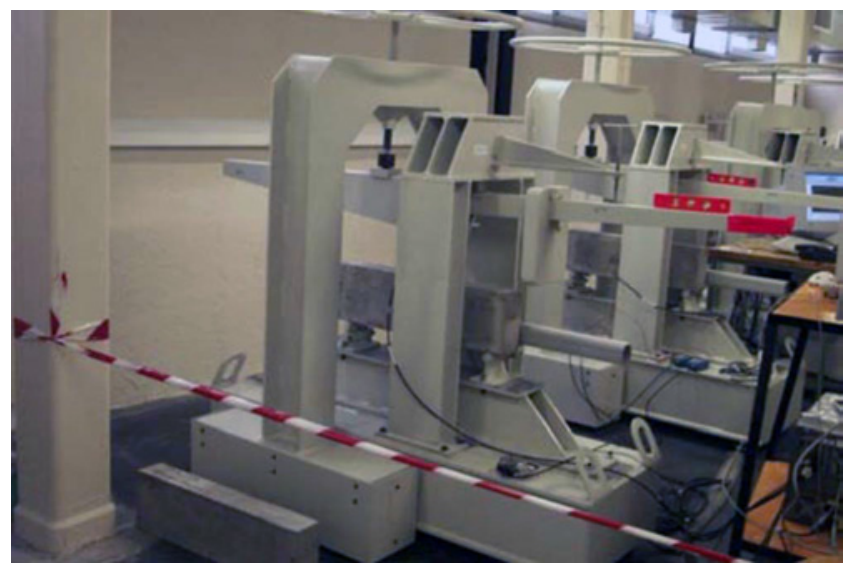

Fig. 4. General view of creep frames

could be measured only. The basic creep displacement was determined by subtracting the instantaneous elastic displacement from the total displacement.

The results in Fig. 6 show that increasing the applied load increases the basic displacement creep magnitude. For the two lower loading levels, creep develops very fast in the first days of loading and stabilizes after a few weeks. For the specimen loaded at $80 \%$ of the maximum load, there is a deviation of the creep curve compared to the previous two. After the initial period where creep is growing quickly, the basic displacement creep rate is much higher. Instead of the very moderate growth of the creep displacement that is observed after 20 to 30 days and onwards for the two lower load levels, the test data show that the displacement creep is steadily increasing, same as for the unnotched beams; this is typical of tertiary creep where damage seems to be initiated after about 20 to 30 days of accumulation of local tensile strain in the beam.

Fig. 7 shows the responses obtained for specimens of three different sizes loaded at $80 \%$ of their maximum (instantaneous) capacity. It is clearly highlighted that the basic displacement creep kinetics depends on the size of the specimen. The larger the specimen size, the greater the creep displacement rate, although the specimens carry, proportionally to their size the same maximum stress. Even if they carry the same maximum tensile stress on the bottom face of the specimen or at the notch tip, the stress gradient is much higher in the small specimen compared to the

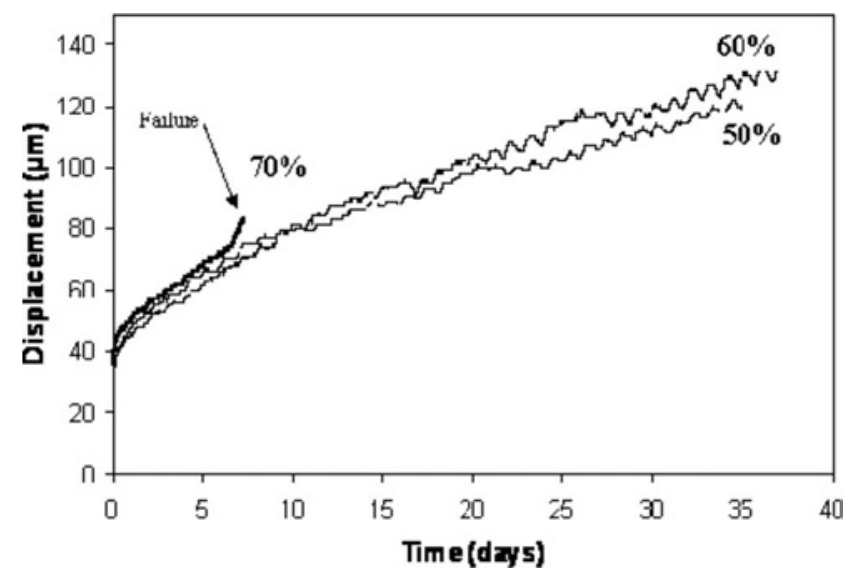

Fig. 5. Deflection of unotched beams $D 1$ for different load levels

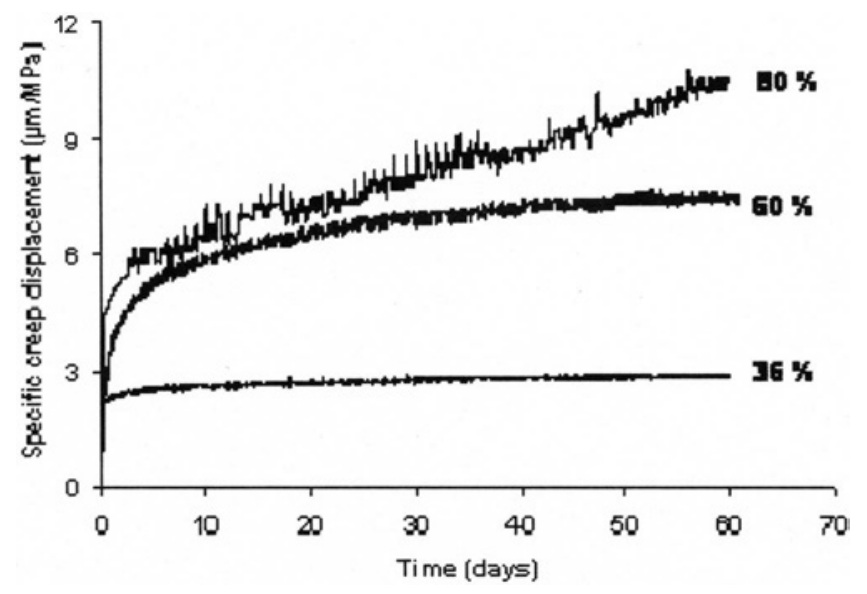

Fig. 6. Basic creep displacement of notched beams $D 1$ for 36,60 , and $80 \%$ of peak load

large one. Consequently, the size of the part of the beam in which the stress is above any given arbitrary value is larger in the large specimens than in the small specimens, and that may generate more creep in large specimens compared to small ones. This may be the explanation for the size dependence of basic displacement creep.

It can also be concluded from the above results that above a threshold load, tertiary creep is observed. Microcracking due to the applied load starts to initiate and modifies the creep rate. Therefore, acoustic emission analysis should be capable of detecting events during creep.

\section{Acoustic Emission in Basic Creep Tests}

Acoustic emission (AE) occurs when there is a sudden change in energy (energy release), due to flaws and defect nucleation or propagation in a specimen. Most studies have focused on relating acoustic emission characteristics to the properties of the fracture process zone (Maji and Shah 1988), and using AE source location analysis to evaluate damage localization (Berthelot and Robert 1987; Haidar et al. 2005; Granger et al. 2007). We applied this technique to the creep tests presented above. The primary aim of this $\mathrm{AE}$ analysis was to illustrate the correlation between the basic displacement creep and the total number of acoustic events. In

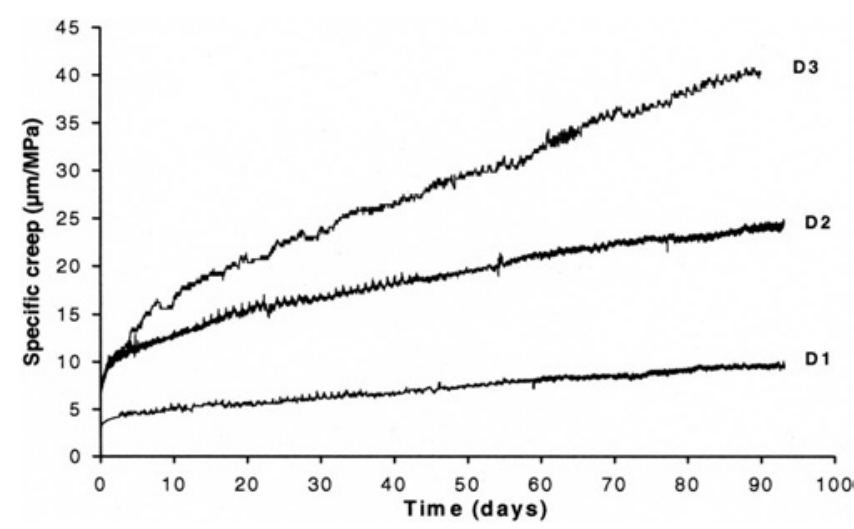

Fig. 7. (a) Basic creep displacement; (b) specific creep of notched beams at $80 \%$ of peak load 


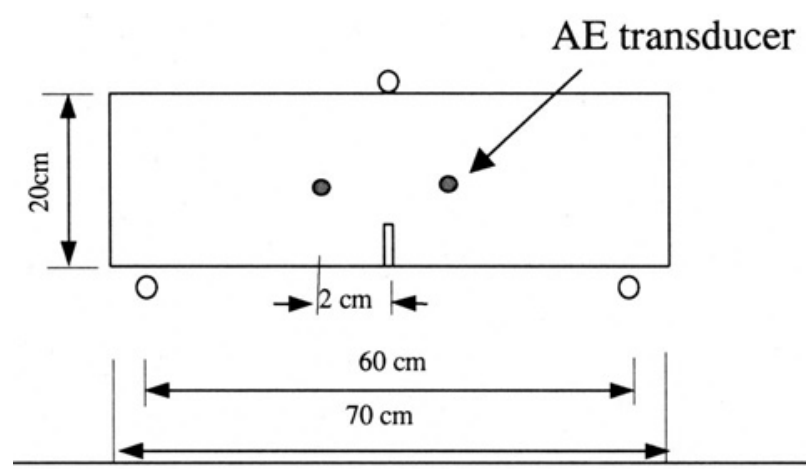

Fig. 8. Specimen geometry and AE transducers position

other terms, our intent was to the qualitative relationship between basic displacement creep strain and damage occurring inside the material.

The AE system used is comprised of a MISTRAS system manufactured by Euro Physical Acoustic Corporation. Piezoelectric transducers $(\mathrm{R} 15 / \mathrm{C}$, resonance frequency of $150 \mathrm{kHz})$ were used. Two transducers were placed nearby the expected location of the process zone, on one side of the specimen. Their horizontal spacing was $40 \mathrm{~mm}$ (centered at the notch location) and they were glued 0,30 , and $100 \mathrm{~mm}$ below the neutral axis of the $D 1, D 2$, and $D 3$ specimens, respectively (Fig. 8). From the data recorded by the transducers, it was possible to compute the horizontal position of each event (but not its location within the depth of the beam because four transducers would be needed). Hence, the results show the projection of all events during creep onto a horizontal line materialized by the transducers. The accuracy of the algorithm, which localizes the acoustic events, was tested with a Hsu-Nielsen source. It is less than or equal to 0.25 times the maximum aggregate size.

The line along which the $\mathrm{AE}$ events were localized was divided into segments of length $5 \mathrm{~mm}$ (approximately the event localization error) and the number of events located in each segment has been counted. The result of this procedure, which is similar to the one used by Haidar et al. (2005) to characterize the size of the fracture process zone (FPZ) in fracture tests, is a set of profiles of cumulated number of events at different times during creep.

Fig. 9 shows these profiles for the small- and medium-sized specimens loaded at $80 \%$ of their maximum capacity during the first 8 days when the acoustic activity is most significant. It is observed that the AE events increase as basic displacement creep develops. During the first days of loading, some moderate acoustic activity is recorded. Fig. 10 shows the cumulated acoustic energy, which becomes almost constant after 8 days approximately, as well as the basic creep displacement rate (Fig. 7). The beams creep without any noticeable acoustic activity. In the primary phase, shortly after the application of the load, the AE activity may be representative of some rearrangement of the microstructure of the material, probably due to microcracking, sliding, or release of internal stresses. In the second phase, creep develops without any AE activity. In this regime, creep is linear and the material is viscoelastic. The results show that such a material response develops without energy released and converted into $\mathrm{AE}$ events.

During the testing program, several large size beams loaded at $80 \%$ of their residual capacity failed before the end of the creep period. As a matter of fact, about $1 / 3$ of such beams survived to

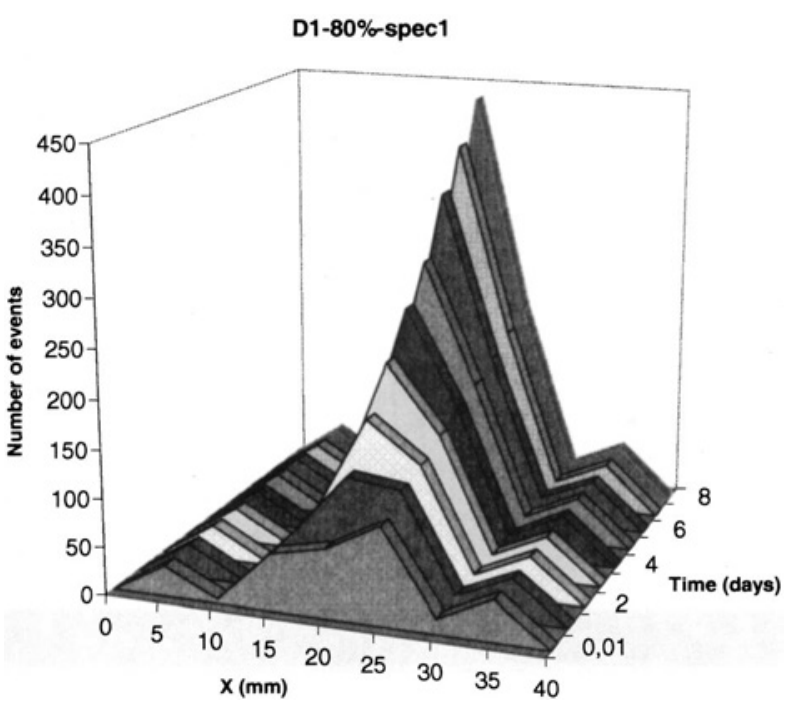

Fig. 9. Profile of the acoustic events recorded during creep for the small- and medium-sized specimen

a creep duration of 90 days. Fig. 11 shows the evolution of the profiles of acoustic events for one of the large specimens that failed after 7.5 days of creep. Note that the number of cumulated events is about four times what was observed in Fig. 9 for the small- and medium-sized specimens (where no failure was observed). These profiles are very similar to those obtained by Haidar et al. (2005) on three-point bend fracture tests. They show that due to creep, microcracking is initiated after a certain accumulation of creep strain and this damage initiation may yield to failure when the applied load is sufficiently high. AE event analysis shows clearly that there is a coupling between creep and damage.

\section{Residual Capacity Tests}

Whenever damage occurs during the creep phase, the residual capacity of the beam should be modified, and it is the purpose of this section to investigate this effect. After 90 days of loading, the beams subjected to $80 \%$ of the maximum load were removed from the creep frames and then immediately subjected to threepoint bending loading up to failure with a constant loading rate.

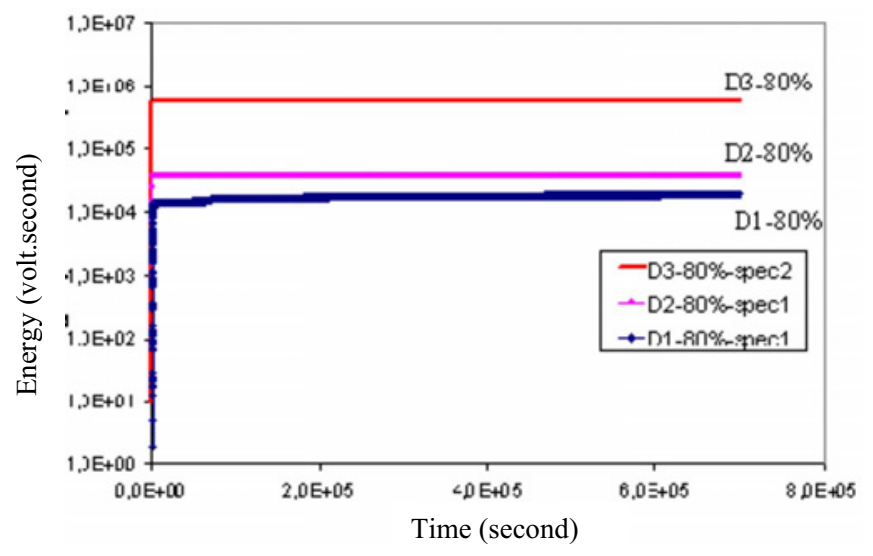

Fig. 10. Evolution of the cumulated energy of acoustic events during the creep tests 


\section{D3-80\%-spec1}

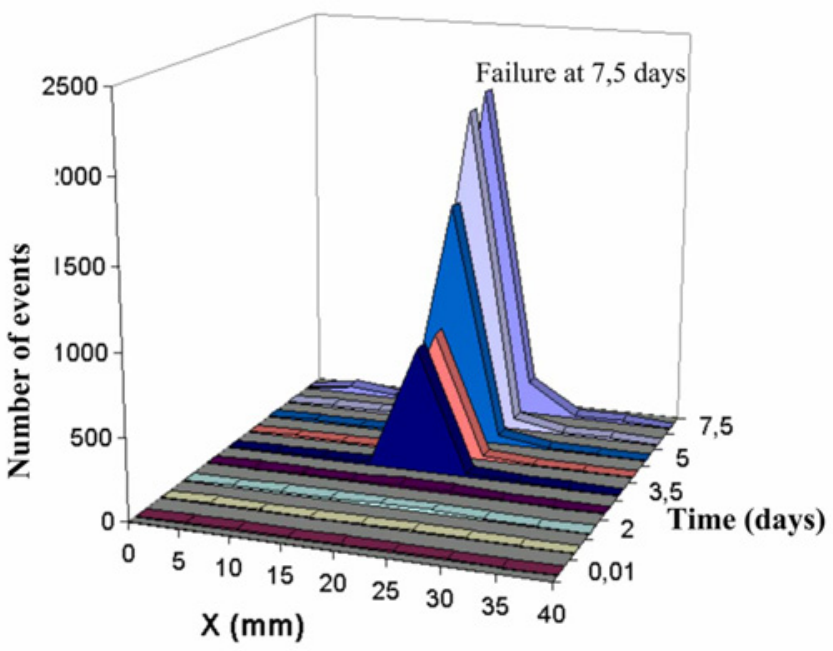

Fig. 11. Profiles of acoustic events for one of the largest specimens, which failed after 7.5 days as a function of time

Comparison specimens cast at the same time as those subjected to creep were kept under the same conditions of temperature and relative humidity. Those specimens were tested at the same time. The experiments are similar to those described in the section "Creep Tests" of this paper. For each specimen size $(D 1, D 2, D 3)$, at least three specimens and three comparison specimens have been tested.
Fig. 12 shows the average load-deflection curves obtained before and after creep. Note again that for the larger specimens, a large majority of them failed during the creeping phase. Therefore, the average curve should be looked at as an upper bound of the residual capacity of the beams after creep and not as an average response. For the small and medium size beams, the difference between the specimens subjected to creep and the comparison ones is small. The postpeak responses are almost the same (the medium-sized specimen looks a bit more ductile after creep). There is a slight decrease of the average peak load for the medium-sized specimen, but it is within the experimental dispersion. The standard deviation of the test data after (and before) creep is $9 \mathrm{~N}$ for the small specimens, $470 \mathrm{~N}$ for the medium ones, and $2,700 \mathrm{~N}$ for the large ones. For the large beams, the same postpeak responses seem to be recovered and at the same time, there is a decrease of the peak load, which is slightly above the experimental dispersion. Again, recall that in the case of large beams, this peak value should be looked at as an upper bound. Many specimens failed under creep at a load of about $19 \mathrm{kN}$, below the observed peak load for the specimen tested after creep on this figure. Finally, notice also that the instantaneous stiffness of the beams does not seem to be influenced by creep.

The difference on the load-deflection curves is very slight in Fig. 12; the same as for the case of chemical leaching coupled to mechanical damage (Le Bellégo et al. 2003); this difference can be enlarged using the size effect law, that is when the extrapolation to specimens of larger and larger sizes is considered. A simple version of Bazant's size effect law is used (Bazant and Planas 1998). The nominal strength (MPa) is obtained with the formulas

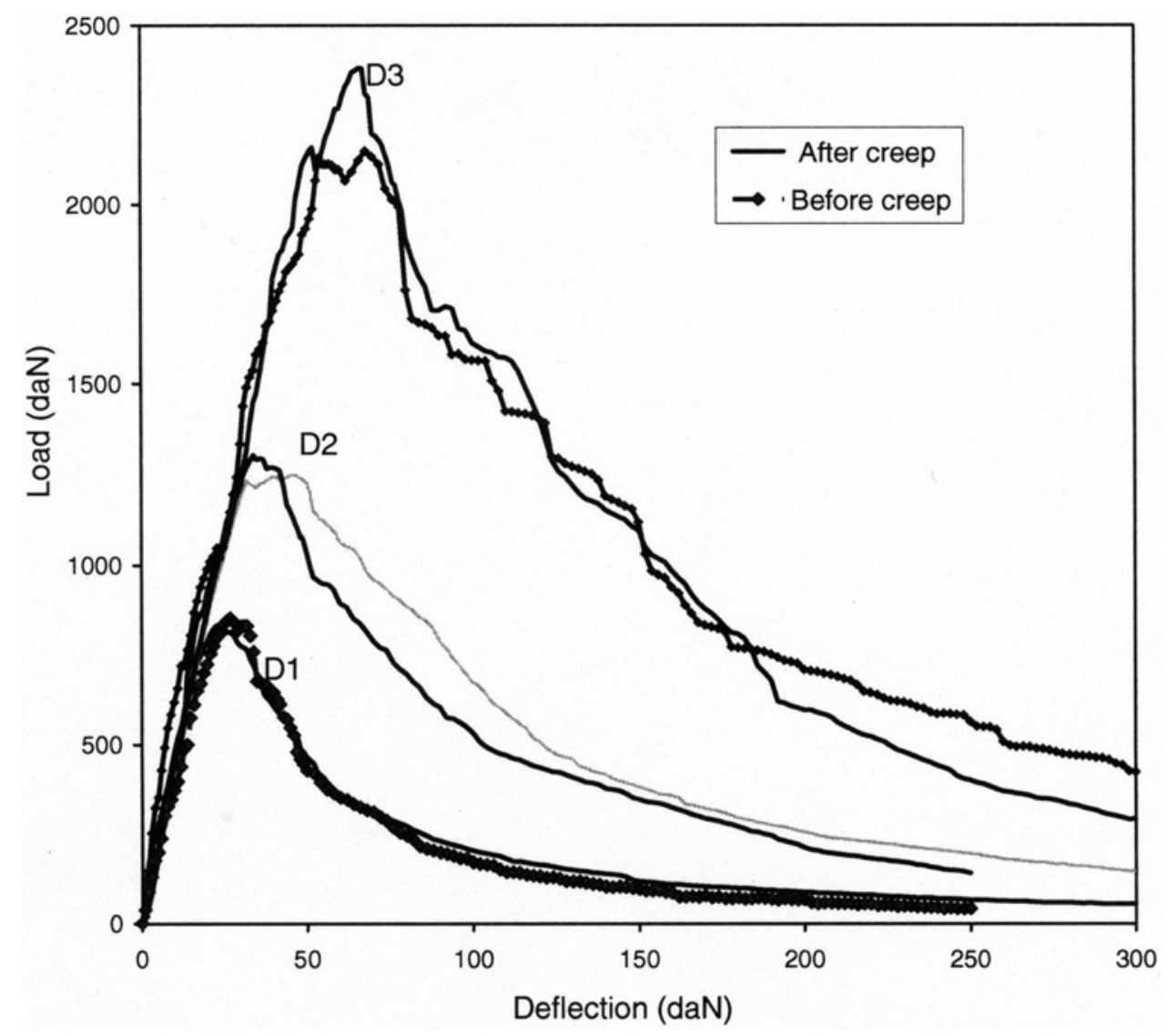

Fig. 12. Mechanical response during fracture tests before and after creep 


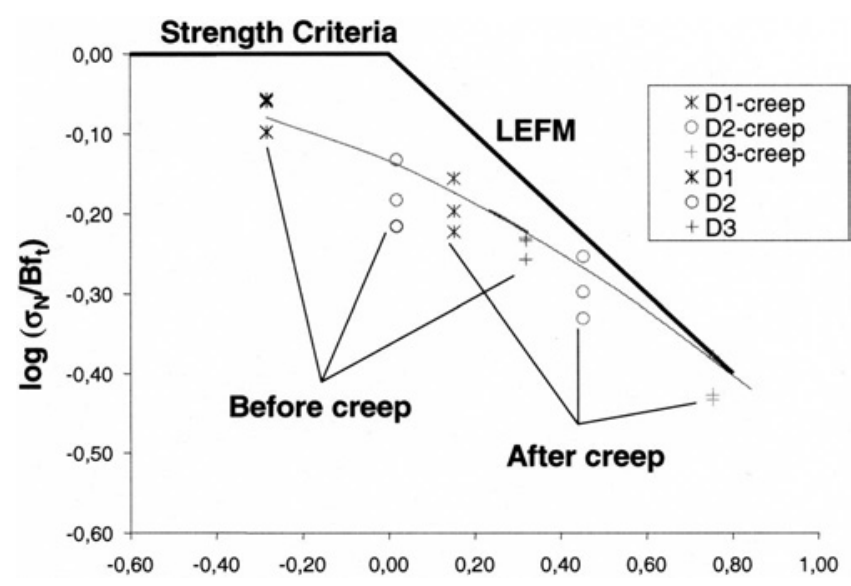

Fig. 13. Size effect test data before and after creep

$$
\sigma_{N}=\frac{3}{2} \frac{\mathrm{Fl}}{b(0.9 D)^{2}}
$$

and

$$
\sigma_{N}=\frac{\mathrm{Bf}_{t}}{\sqrt{1+D / d_{o}}}
$$

where $b=$ thickness of the beam; $D=$ height $(\mathrm{mm}) ; l=\operatorname{span}(\mathrm{mm})$; $F=$ maximal load $(\mathrm{N}) ; f_{t}=$ tensile strength of the material; $d_{o}$ $=$ characteristic size that corresponds to a change of failure mechanism between strength of materials and linear elastic fracture mechanics; and $B=$ geometry related parameter. $D / d_{o}$ can be considered as a measure of the structural brittleness. The larger it is, the more brittle the structure.

Fig. 13 shows the size effect test data for the two sets of beams, with and without creep. We can observe a shift of the data points to the right when creep is applied prior to the fracture tests. Upon creep, the behavior of the beams is closer to linear elastic fracture mechanics. It is then interesting to compare the parameters obtained from the size effect law fitted before and after creep. The size effect law provides the value of $d o$, but also the size of the FPZ $C_{f}$ at failure (peak load), the fracture energy $G_{f}$ extrapolated for a specimen of infinite size, and finally Hillerborg's characteristic length $L_{\mathrm{ch}}$

$$
L_{\mathrm{ch}}=\frac{\mathrm{EG}_{f}}{f_{t}^{2}}
$$

The comparison is shown in Table 3. We may observe that there is a small increase of the tensile strength (which should be within the experimental dispersion observed on this parameter and measured according to the size effect method). Most importantly, there is a $60 \%$ decrease of the length of the FPZ, the fracture energy is divided by 2 , and the characteristic length as well. This test series shows that upon creep, at a sufficiently high load level, the fracture characteristics decrease. Due to creep, some damage develops and reduces the fracture properties of the material.

Table 3. Variation of the Size Effect Parameters due to Creep

\begin{tabular}{lccccc}
\hline Parameter & Do $(\mathrm{mm})$ & $\mathrm{B} * \mathrm{ft}(\mathrm{MPa})$ & $\mathrm{Gf}(\mathrm{N} / \mathrm{m})$ & $\mathrm{Cf}(\mathrm{mm})$ & Lch $(\mathrm{mm})$ \\
\hline Before creep & 198 & 6.5 & 33.1 & 31 & 100 \\
After creep & 72 & 9 & 18.7 & 11.5 & 46.3 \\
\hline
\end{tabular}

The above results are consistent with experimental data by Gettu and Bazant (1992) for specimens tested at several rates of loading. The lower the loading rate, the more brittle the specimen, with the same shift toward linear elastic fracture mechanics (LEFM) on the size effect plot. Theoretically, one explanation for this increase is the relaxation of stresses in the FPZ, nearby the notch. The relaxation of the stress in the FPZ yields a decrease of the FPZ size (and length) of brittleness, as shown theoretically from discrete modeling and avalanche statistics by Baxevanis et al. (2005). This decrease of the FPZ size induces a shift toward LEFM of size effect test data. There is another explanation based on the decrease of the activation energy needed for failure due to creep (Bazant and Li 1997). Some recent numerical investigations by Baxevanis et al. (2006), show also that this shift of the size effect law may be described with a linear viscoelastic model coupled to nonlocal damage. The coupling between damage and creep follows the same technique as in Omar et al. (2004).

\section{Conclusions}

The following conclusions can be drawn from the above test results:

- For high load levels, tertiary creep, i.e., a viscoelastic response of the material coupled with an evolution of damage, occurs. In our bending tests, tertiary creep is observed for load levels that are above $70 \%$ of the maximum carrying capacity of the beam subjected to monotonically increasing loads. This limit is consistent with other experimental data, namely, in compression tests (Mazzotti and Savoia 2003).

- Basic displacement creep on bending beams is size dependent. The rate of creep increases with the specimen size.

- AE shows that there is some activity during the initial creep phase, shortly after the application of the load. For the small specimens, the AE activity then vanishes during linear creep. In the tertiary phase of creep, it starts again and clearly corresponds to the growth of damage prior to failure. This damage is due to the accumulation of creep strain. It is an indication that damage should be coupled to creep.

- Residual capacity tests show that displacement creep has an influence on the residual carrying capacity of the beams. The fracture energy decreases, and at the same time the FPZ decreases too.

These test results now have to be used for the development of a constitutive relation in which creep and damage are coupled. From a practical point of view, this decrease of the residual capacity of the beams due to creep may have some consequences: it may reduce the safety margin of structural components with respect to accidental loads, even if during their service life they are subjected to moderate loads (although above the threshold where damage appears). Some models exist in the literature [see, for instance, Mazzotti and Savoia (2001); Mazzotti and Savoia (2003); Zi and Bazant (2001); Bazant and Jirasek (1992); Omar et al. (2004); Benboudjema et al. (2005)], and should be compared with the above results as well. Furthermore, additional test data dealing with larger sizes of specimen, higher load levels in basic creep experiments, followed by size effect tests are expected to provide a more comprehensive understanding of the creep damage interaction and of the influence of creep on the fracture energy of concrete. 


\section{Acknowledgments}

Financial support from the European Commission through the MAECENAS project (FIS5-2001-00100) and from the partnership between Electricité de France and the R\&DO Group through the MECEN project are gratefully acknowledged.

\section{References}

Baxevanis, T., Dufour, F., and Pijaudier-Cabot, G. (2005). "Interface crack propagation in porous and visco-elastic materials analysed with discrete models." Int. J. Fract., 141, 561-571.

Baxevanis, T., Dufour, F., Pijaudier-Cabot, G., and Desiassyifaianty R. (2006). "Bifurcation and size effect in a visco-elastic non local damageable material." Proc., EURO-C, N. Bicanic et al., eds., Balkema, Rotterdam, The Netherlands, 283-290.

Bazant, Z. P., and Jirasek, M. (1992). "R-curve modeling of rate effect in static fracture and its interference with size effect." Fracture mechanics of concrete structures, Proc., Int. Conf. Fracture Mechanics of Concrete Structures, Elsevier Applied Science, London, 918-923.

Bazant, Z. P., and Li, Y. N. (1997). "Cohesive crack with rate dependent opening and viscoelasticity: I. Mathematical model and scaling." Int. J. Fract. 86, 247-265.

Bazant, Z. P., and Planas, J. (1998). "Fracture and size effect in concrete and other quasibrittle materials." CRC, Boca Raton, Fla.

Benboudjema, F., Meftah, F., and Torrenti, J.-M. (2005) "Interaction between drying, shrinkage, creep and cracking phenomena in concrete." Eng. Struct., 27, 39-250.

Berthelot, J. M., and Robert, J. L. (1987). "Modelling concrete damage by acoustic emission." J. Acoustic Emission 7, 43-60.

de Borst, R., van den Boogaard, A. H., and Sluys, L. J. (1993). "Computational issues in time-dependent deformations and fracture of concrete." Proc., 5th Int. RILEM Symp. on Creep and shrinkage of Concrete, 309-325.

Gettu, R., and Bazant, Z. P. (1992). "Rate effects and load relaxation: Static fracture of concrete." ACI Mater. J., 89(5), 456-468.

Granger, S., Loukili, A., Pijaudier-Cabot, G., and Chanvillard, G. (2007).
"Experimental characterization of the self-healing of cracks in an ultra high performance cementitious material: Mechanical tests and acoustic emission analysis." Cem. Concr. Res., 37, 519-527

Haidar, K., Pijaudier-Cabot, G., Dubé, J. F., and Loukili, A. (2005). "Correlation between internal length, fracture process zone and size effect in mortar and model materials." Mater. Struct., 38, 201-210.

Khelidj, A., Loukili, A., Bastian, G. (1998). "Experimental study of the hydro-chemical coupling inside maturing concretes: Effect on various types of shrinkage." Mater. Struct., 31, 588-594.

Le Bellégo, C., Pijaudier-Cabot, G., Gérard, B., Dubé, J. F., and Molez, L. (2003). "Coupled mechanical and mechanical damage in calcium leached cementitious structures." J. Eng. Mech., 129, 3 333-341.

Maji, A. K., and Shah, S. P. (1988). "Process zone and acoustic emission in concrete." Exp. Mech., 28, 27-33.

Mazars, J., and Pijaudier-Cabot, G. (1996). "From damage to fracture mechanics and conversely: A combined approach." Int. J. Solids Struct., 33, 3327-3342.

Mazzotti, C., and Savoia, M. (2001). "Experimental study of non-linear creep of concrete at high stress level." Proc., CONCREEP-6, F. J. Ulm et al., eds., Elseiver, London, 239-243.

Mazzotti, C., and Savoia, M. (2003). "Nonlinear creep damage model for concrete under uniaxial compression.” J. Eng. Mech., 129, 9, 10651075.

Omar, M., Pijaudier-Cabot, G., and Loukili, A. (2004). "Etude comparative du couplage endommagement-Fluage." Revue Francaise Genie Civil, 8, 457-482.

RILEM. (1990). "Size-effect method for determining fracture energy and process zone size of concrete." Mater. Struct., 23, 461-465.

Rüsch, H., Sell, R., Rasch, C., and Stöckl, S. (1958). "Investigations into the strength of concrete under sustained load." Proc., RILEM Symp. on the Influence of Time on the Strength and Deformation of Concrete.

Spinner, S., and Teft, W. E. (1961). "A method for determining mechanical resonance frequencies and for calculating elastic modulus from these frequencies." Proc., Amer. Soc. Testing Mat., 61, 1221-1238.

Zi, G., and Bazant, Z. P. (2001). "Continuous relaxation spectrum of concrete creep and its incorporation into microplane model M4." Proc., CONCREEP-6, F. J. Ulm et al., eds., Elsevier, London, 239243. 